\title{
ORIGINAL ARTICLE \\ Autologous incubated macrophage therapy in acute, complete spinal cord injury: results of the phase 2 randomized controlled multicenter trial
}

\author{
DP Lammertse ${ }^{1,2}$, LAT Jones ${ }^{3}$, SB Charlifue ${ }^{1}$, SC Kirshblum ${ }^{4,5}$, DF Apple ${ }^{6}$, KT Ragnarsson ${ }^{7}$, SP Falci ${ }^{8}$, \\ RF Heary ${ }^{9}$, TF Choudhri ${ }^{10}$, AL Jenkins ${ }^{10}$, RR Betz ${ }^{11}$, D Poonian ${ }^{12}$, JP Cuthbert ${ }^{1}$, A Jha ${ }^{1,2}$, \\ DA Snyder ${ }^{13}$ and $\mathrm{N}$ Knoller ${ }^{14}$
}

Study design: Randomized controlled trial with single-blinded primary outcome assessment.

Objectives: To determine the efficacy and safety of autologous incubated macrophage treatment for improving neurological outcome in patients with acute, complete spinal cord injury (SCl).

Setting: Six SCI treatment centers in the United States and Israel.

Methods: Participants with traumatic complete SCI between C5 motor and T11 neurological levels who could receive macrophage therapy within 14 days of injury were randomly assigned in a 2:1 ratio to the treatment (autologous incubated macrophages) or control (standard of care) groups. Treatment group participants underwent macrophage injection into the caudal boundary of the $\mathrm{SCl}$. The primary outcome measure was American Spinal Injury Association (ASIA) Impairment Scale (AIS) A-B or better at $\geqslant 6$ months. Safety was assessed by analysis of adverse events (AEs).

Results: Of 43 participants ( 26 treatment, 17 control) having sufficient data for efficacy analysis, AIS A to B or better conversion was experienced by 7 treatment and 10 control participants; AIS A to C conversion was experienced by 2 treatment and 2 control participants. The primary outcome analysis for subjects with at least 6 months follow-up showed a trend favoring the control group that did not achieve statistical significance $(P=0.053)$. The mean number of AEs reported per participant was not significantly different between the groups $(P=0.942)$.

Conclusion: The analysis failed to show a significant difference in primary outcome between the two groups. The study results do not support treatment of acute complete $\mathrm{SCl}$ with autologous incubated macrophage therapy as specified in this protocol.

Spinal Cord (2012) 50, 661-671; doi:10.1038/sc.2012.39; published online 24 April 2012

Keywords: acute spinal cord injury; neurological recovery; macrophage; cellular therapy

\section{INTRODUCTION}

Although clinicians involved in the management of spinal cord injury (SCI) have been encouraged by the increasing number of bench-tobedside translations, which have led to clinical trials, a consensus standard of care for improving neurological outcomes has yet to be established. ${ }^{1-3}$ Within this context, the ongoing effort to find promising new treatments has included investigation of the immune system response to injury as a therapeutic target. Specifically, study of the macrophage immune response to injury in the poorly-regenerative adult rodent central nervous system has been shown to be blunted and delayed compared with that in the more robustly regenerative peripheral nervous system. ${ }^{4,5}$ Manipulation of macrophage activation by monocyte co-incubation with peripheral nervous system tissue was initially studied in a rodent optic nerve transection model, which showed improved regeneration of nerve fibers exposed to macrophages activated with sciatic nerve. ${ }^{6}$ The study of peripheral nerve-activated macrophages in a rat spinal cord transection model followed, showing partial behavioral and electrophysiological recovery of hind limb motor function as well as immunohistochemical evidence of nerve fiber continuity across the lesion site. ${ }^{7}$ A more clinically relevant model was studied with skinactivated macrophages injected into contused rat spinal cord that resulted in improved motor recovery and reduced spinal cyst formation. ${ }^{8}$ This study also identified macrophage characteristics associated with the activation paradigm, finding elevated macrophage expression of cell surface molecules characteristic of antigenpresenting cells as well as macrophage secretion of interleukin-1 $\beta$ and brain-derived neurotrophic factor. The authors posited that co-incubation of monocytes with excised skin (an injured tissue with regenerative capacity) produces macrophages with an

${ }^{1}$ Department of Research, Craig Hospital, Englewood, CO, USA; ${ }^{2}$ Department of Physical Medicine and Rehabilitation, University of Colorado Denver, Aurora, CO, USA; ${ }^{3}$ SCI Clinical Trial Consultant, Boulder, CO, USA; ${ }^{4}$ Department of Physical Medicine and Rehabilitation, University of Medicine and Dentistry of New Jersey, Newark, NJ, USA; ${ }^{5}$ Kessler Institute for Rehabilitation, West Orange, NJ, USA; ${ }^{6}$ Shepherd Center, Atlanta, GA, USA; ${ }^{7}$ Department of Rehabilitation Medicine, Mount Sinai Medical Center, New York, NY, USA; ${ }^{8}$ Department of Neurosurgery, Craig Hospital, Englewood, CO, USA; ${ }^{9}$ Department of Neurological Surgery, University of Medicine and Dentistry of New Jersey, Newark, NJ, USA; ${ }^{10}$ Department of Neurosurgery, Mount Sinai Medical Center, NY, USA; ${ }^{11}$ Department of Orthopedics, Shriners Hospital for Children, Philadelphia, PA, USA; ${ }^{12}$ DP Clinical, Rockville, MD, USA; ${ }^{13}$ Gamida Cell Ltd., Jerusalem, Israel and ${ }^{14}$ Department of Neurosurgery, Sheba Medical Center, Tel Hashomer, Israel Correspondence: Dr DP Lammertse, Medical Director of Research, Craig Hospital, 3425 South Clarkson Street, Englewood, CO 80113-2811, USA Email: dlammertse@craighospital.org

Received 19 September 2011; revised 9 March 2012; accepted 14 March 2012; published online 24 April 2012 
'alternatively activated' wound-healing phenotype. This beneficial phenotype would not only provide direct benefit by removal of growth-inhibitory myelin components from the cellular environment and the potential secretion of trophic factors, but would also provide indirect benefit through cytokine signaling and activation of the local adaptive immune response. ${ }^{8}$

These pre-clinical findings led to an open-label phase 1 clinical trial of autologous incubated macrophages for treatment of patients with acute complete C5-T11 SCI. ${ }^{9}$ That trial enrolled eight participants with complete SCI who were treated with autologous macrophages prepared by co-incubating peripheral blood monocytes with harvested autologous skin. A total dose of 4 million macrophages was introduced directly into the spinal cord within 2 weeks of SCI by four separate injections at the caudal boundary of the contusive lesion (bilateral $20 \mu \mathrm{l}$ injections into lateral white matter, bilateral $10 \mu \mathrm{l}$ injections into the dorsal columns). The participants were followed for 12 months with serial neurological examinations and clinical follow-up of adverse events (AEs). During the study period, 3 of 8 subjects (37.5\%) exhibited improvement in their American Spinal Injury Association (ASIA) Impairment Scale (AIS) grade from A (complete injury) to $\mathrm{C}$ (sensory and motor incomplete). AEs included several categorized as severe (pulmonary embolism in two patients, osteomyelitis in one patient) and a list of other AEs that are commonly seen in patients hospitalized with SCI. With these results showing a higher recovery rate than that commonly reported in the literature and an $\mathrm{AE}$ experience that did not suggest significant safety concerns, the present study was initiated in 2003 as a phase 2 multicenter randomized controlled trial (RCT) of autologous incubated macrophage therapy for acute complete SCI-the first RCT of a cell-based intervention for patients with acute SCI. This project studied the hypothesis that treatment with autologous incubated macrophages introduced into the spinal cord within 14 days of SCI would be associated with improved neurological outcomes and reasonable safety. The logistics of conducting a rigorous clinical trial of a cell-based surgical intervention within the short time lines of acute SCI treatment were challenging and have been reported separately. ${ }^{10}$ Here we report the efficacy and safety findings of the phase 2 multicenter trial.

\section{METHODS}

\section{Study design and setting}

This study was a phase 2 multicenter randomized parallel group controlled trial comparing autologous incubated macrophage treatment with standard care utilizing single-blinded outcome assessment of the primary and secondary endpoints. Participants were enrolled in one of the six SCI centers in the United States and Israel (Table 1).

\section{Participants}

The trial enrolled participants with acute SCI who could receive the study intervention within 14 days of injury and were determined, based on the International Standards for Neurological Classification of Spinal Cord Injury (ISNCSCI) $^{11}$ to have neurologically complete injuries (AIS A) between C5 motor/C4 sensory level and T11 neurological level. The complete list of inclusion/exclusion criteria are listed in Table 2, several of which are related to the study intervention and thus deserve additional comment. The 2-week treatment window was chosen based on unpublished preclinical rat studies that showed lack of response when the macrophage intervention was delayed 14 and 21 days after injury. The blood transfusion exclusion was intended to insure that only autologous monocytes were utilized in the treatment. Patients with hemoglobin levels of $<8.5 \mathrm{~g} \mathrm{dl}^{-1}$ were excluded because of the need for a $250 \mathrm{ml}$ blood harvest for cell processing, which would have aggravated the existing anemia before major surgery. Patients with a history of chronic
Table 1 Participating Study Centers

\begin{tabular}{ll}
\hline Study Center & Location \\
\hline Chaim Sheba Medical Center & Tel Hashomer, Israel \\
Craig Hospital & Englewood, Colorado \\
Mount Sinai School of Medicine/Mount Sinai Hospital & New York, New York \\
Kessler Medical Rehabilitation Research Corporation/ & West Orange/Newark, \\
University of Medicine and Dentistry New Jersey & New Jersey \\
Shriners Hospital & Philadelphia, \\
& Pennsylvania \\
Shepherd Center & Atlanta, Georgia \\
\hline
\end{tabular}

treatment with immune modulating drugs or injury-related steroid treatment within 5 days of tissue harvest were excluded because of concerns that such treatment might interfere with macrophage activation during cell processing. Patients on concomitant treatment with erythropoietin were excluded because of unpublished experience, suggesting an increased likelihood of cell processing batch failure. Contusion length $>3 \mathrm{~cm}$ measured by magnetic resonance imaging (MRI) was an exclusion intended to eliminate subjects less likely to respond to the study intervention. This was based on the phase 1 trial experience that 'responders' (subjects who became neurologically incomplete during the study period) had contusion lengths of $\leqslant 3 \mathrm{~cm}$. The contusion length was determined by software 'ruler' assessment of the rostral-caudal extent of blood product signal within the spinal cord; that is, not a measurement of edema signal.

Patients identified as being potentially eligible were approached to determine interest in trial participation. Those who wished to participate completed informed consent and were enrolled. After screening assessment of demographic, neurological, laboratory and MRI variables was performed, those who met eligibility criteria were randomly assigned in a 2:1 ratio to either the treatment or control groups, respectively.

\section{Randomization}

Centralized computer-generated randomization tables with block size of nine participants were used to determine assignment within the study center. The block size was not known by the study center personnel. The randomization scheme was designed to produce a 2:1 ratio of treatment to control group assignment by center. Sequentially numbered sealed envelopes containing designation of treatment or control were maintained at each study center and opened in sequence after confirmation of eligibility to reveal group assignment. Participants assigned to the control group received standard-of-care treatment for SCI. Participants assigned to the treatment group received standard-of-care treatment for SCI as well as administration of autologous incubated macrophages. On the basis of practical and ethical considerations, incorporation of a sham intervention procedure was not undertaken in this trial; hence, the participants were aware of their group assignment from the time of randomization. ${ }^{10}$

\section{Intervention}

Preparation of macrophages for use in the treatment group participants began with autologous skin and blood harvest. Approximately $230 \pm 20 \mathrm{ml}$ of peripheral blood was withdrawn for cell processing. Autologous skin was harvested as a $12 \times 3 \mathrm{~cm}$ diamond-shaped full thickness excision of the dermal layer from the medial surface of either arm. The harvested blood and skin were delivered to a specified Cell Center certified by the study sponsor to manufacture autologous incubated macrophage therapy according to Good Manufacturing Practice. The details of cell processing have been previously described. ${ }^{9}$ An enriched monocyte fraction was obtained by centrifugation on density gradients. These cells were co-incubated with decontaminated skin segments for 1 day with the resulting cell preparation being assessed for release criteria that verified suitability for transplantation based on characteristics including viability $(\geqslant 80 \%)$ and purity $\left(\geqslant 60 \% \mathrm{CD} 14^{+}\right.$macrophages) listed in Table 3. One and a half million cells were suspended in $120 \mu \mathrm{l}$ of medium and 
Table 2 Inclusion/exclusion criteria

\section{Inclusion criteria}

1. Traumatic $\mathrm{SCl}$ (within 14 days).

2. Age between 16 and 65 inclusive.

3. Complete spinal cord injury (AIS grade A).

4. A single spinal cord lesion between $\mathrm{C} 5$ motor level and $\mathrm{T} 11$ neurological level by the ISNCSCI.

5. The location and size of the injury can be determined by magnetic resonance imaging (MRI).

6. Informed consent obtained and informed consent form signed.

\section{Exclusion criteria}

1. Patients $>14$ days after injury.

2. Women who are pregnant or breast feeding.

3. Coma.

4. Gunshot or other penetrating trauma.

5. Fever (temperature above $38.4^{\circ} \mathrm{C}$ ) $24 \mathrm{~h}$ before scheduled tissue harvesting.

6. Blood transfusion within 3 days before scheduled tissue harvest.

7. Ventilator assistance within $24 \mathrm{~h}$ before randomization.

8. Hemoglobin $<8.5 \mathrm{gm} \mathrm{dl}^{-1}$.

9. Anatomic transection of the spinal cord visualized by MRI.

10. Longitudinal dimension of the injury determined by $\mathrm{MRI}>3 \mathrm{~cm}$.

11. Human immunodeficiency virus, hepatitis B or hepatitis $C$ serology positive.

12. Serious pre-existing medical conditions.

13. Any disease, concomitant injury, condition or treatment that interferes with the performance or interpretation of the neurological examinations.

14. Previous or concomitant treatment with:

- Immune modulators or experimental drugs within 60 days before study enrollment.

- Concomitant treatment with immune modulators (that is, dexamethasone, methylprednisolone) within 5 days before skin harvesting.

- Concomitant treatment with hematopoietic inducers (that is, erythropoietin) following injury.

15. Patient is unlikely to be available for follow-up as specified in the protocol.

16. Patient has a condition or has received medical treatment that, in the judgment of the investigator, precludes successful participation in the study.

placed into a $0.3 \mathrm{ml}$ U-100 Microfine syringe with a $30 \mathrm{G}$ fixed needle and sterilely packaged for delivery to the operating room where they were administered to the participant within $4 \mathrm{~h}$ of syringe loading. Accounting for final assessment of release criteria, packaging and documentation, the cells typically were released from the Cell Center to the operating room with $2 \mathrm{~h}$ remaining in the cell expiration window (approaching the end of the trial, additional cell stability studies permitted extending this cell expiration time to $8 \mathrm{~h})$. The mean elapsed time between initiation of cell processing and cell product expiration (the time window by which completion of cell injection was required) was $37 \mathrm{~h} 12 \mathrm{~min}$ (range of $36 \mathrm{~h} 3 \mathrm{~min}-38 \mathrm{~h} 12 \mathrm{~min}$ ). All cell injections were accomplished within the defined time window.

Surgical administration of the autologous incubated macrophages was performed under general anesthesia with appropriate antibiotic prophylaxis. With the participant in the prone position, a linear midline incision was made over the site of the injury based on preoperative MRI localization. Following bilateral paraspinal muscle separation and retraction, existing surgical instrumentation was removed as necessary for adequate exposure. Laminectomy was then performed to extend one level above and below the injury site. Intraoperative fluoroscopy was used as necessary for confirmation of the injury site referenced to preoperative MRI anatomic landmark correlations. The dura was then opened at the midline and retracted laterally by stay sutures allowing direct visual confirmation of the injury location. The caudal contusion boundary had been identified preoperatively by MRI and correlated with anatomic landmarks for intraoperative localization. At the neurosurgeon's discretion, intraoperative spinal sonography was also utilized for precise identification of the contusion boundaries ( 11 of the 26 procedures), verifying the preoperative MRI-defined localization. Six $20 \mu$ injections, each containing 250000 autologous incubated macrophages (total dose of $1.5 \times 10^{6}$ cells in $120 \mu \mathrm{l}$ ), were performed with a single hand-held syringe at the caudal boundary of the spinal cord contusion. Risk/benefit considerations prompted the choice of injection location based on unpublished preclinical rat studies demonstrating that optimal effect could be achieved with macrophages
Table 3 Autologous incubated macrophage release criteria

\begin{tabular}{|c|c|}
\hline Test & Release criterion \\
\hline Gram stain & Negative \\
\hline Bacterial culture & Negative interim culture \\
\hline Endotoxin units & $\leqslant 1.66$ Endotoxin units $\mathrm{ml}^{-1}$ \\
\hline Cell viability & $\geqslant 80 \%$ \\
\hline Cell granularity & $25 \%$ of cells contain $\geqslant 4$ granules \\
\hline Cell morphology & Relatively large, irregular, granulated cells \\
\hline Culture purity $\left(\mathrm{CD} 14^{+}\right.$cells) ${ }^{a}$ & $\geqslant 60 \%$ \\
\hline Interleukin- $1 \beta$ secretion & $\geqslant 50 \mathrm{pg}$ per $10^{6} \mathrm{CD} 14^{+}$cells \\
\hline
\end{tabular}

a In addition to CD14 + macrophages, (unpublished) analysis of activated macrophage cell preparation batches show detectable quantities of T lymphocytes (average 9.5\%), NK cells (average $4 \%$ ), B lymphocytes (average $3 \%$ ), basophils (average $2 \%$ ) and traces of neutrophils $(<1 \%)$.

delivered to the caudal contusion boundary and that the addition of rostral injections did not improve the recovery rate. After each injection, the syringe needle was left in place for an additional $20-30$ s to prevent the injected material from exuding out the needle track. Accurate delivery of volume was confirmed in 152 of 156 injections (97.5\%) performed in the 26 participants with only four discrepant injection volumes reported (range 15-30 $\mu$ l). Cell injection material expression from the needle track was documented in only 4 of the 156 injection sites in a single participant. The injections were directed symmetrically into the region of the posterior columns, corticospinal tracts and spinothalamic tracts. Localization coordinates (depth and distance lateral from midline) of the individual injections was determined by surgical judgment and not defined precisely in the protocol. The dura was then closed primarily or with expansion duraplasty at the discretion of the neurosurgeon. 
Instrumentation was then replaced if previously removed and final layers of closure accomplished.

\section{Rehabilitation}

The protocol specified that rehabilitation would be conducted by an interdisciplinary team in a program that meets the accepted standards of care to achieve the outcome goals outlined in the Consortium for Spinal Cord Medicine Clinical Practice Guideline 'Outcomes Following Traumatic Spinal Cord Injury.' ${ }^{12}$

\section{Outcome measures}

The primary efficacy outcome measure was the AIS grade defined by the ISNCSCI. There were three secondary efficacy outcome measures: (1) recovery of two or more motor/sensory levels (in cervical patients) as defined in the ISNCSCI, (2) recovery in motor and sensory total index scores as defined in the ISNCSCI, and (3) recovery of bowel and bladder function by participant self-report questionnaire. Research personnel responsible for performing the ISNCSCI examinations that formed the basis for the primary and secondary endpoints were specifically trained in examination procedures and tested for inter-rater reliability using test volunteers with SCI. The training results for the ISNCSCI motor and sensory outcome examiners in this study have been previously published showing good intra-rater and inter-rater reliability (intraclass correlation coefficient values in the $0.95-1.00$ range) except for sensory scores in individuals with incomplete SCI (where the intraclass correlation coefficients were 0.86 for light touch, 0.69 for pin prick). ${ }^{13}$ Baseline motor and sensory test abnormalities above the neurological level that were the result of non-SCI pathology and that returned to normal during the study period were treated as normal. Similarly, baseline 'not tested' values above the neurological level owing to casting or other immobilization that subsequently tested normal when unrestricted were treated as normal. Baseline 'not tested' sensory dermatomes below the level of injury owing to bandages or halo-vest coverage and so on were treated as absent when subsequent testing confirmed absence of function. The ISNCSCI-based measures were obtained at baseline and at the 1-week, 1-month, 3-month, 6-month, 9-month and 12-month follow-up visits; the bowel and bladder questionnaires were administered at follow-up months 1,6 and 12. Additional exploratory outcome measurements collected included the Functional Independence Measure (FIM) as a measure of functional capacity/burden of care (at day 7 and months 1, 6 and 12), the Craig Handicap Assessment and Reporting Technique (CHART) as a measure of participation (at months 6 and 12), and the Satisfaction With Life Scale (SWLS) as a measure of quality of life (at months 6 and 12). The safety outcome of the trial was defined by the incidence of AEs reported during the study period (collected at baseline, day of study procedures, and all scheduled follow-up visits).

\section{Blinding}

The key primary and secondary outcome measures obtained at 6,9 and 12 months were collected in a single-blinded manner with tape bandaging covering the protocol-defined areas of presumed skin harvest and macrophage injection surgery. During these examinations, participants were instructed not to reveal their group assignment. All primary and secondary outcome measures at the specified time points were successfully obtained in a blinded fashion using this protocol.

\section{Sample size}

The a priori target enrollment for this study was 61 participants based on assumptions of differential AIS grade improvement over 1 year in participants with complete SCI enrolled and treated 7-14 days after injury. The assumptions and other considerations utilized in power calculations that resulted in this enrollment target have been previously reported. ${ }^{10}$ Results from the published phase 1 study ${ }^{9}$ and additional experience from a separate unpublished phase $1 \mathrm{~b}$ study and compassionate use subjects were used to estimate treatment group response. Control group response was estimated for AIS A participants enrolled at 1-2 weeks after injury by analysis of several existing SCI databases (Israeli and the National Spinal Cord Injury Database from the United States). These analyses suggested that the proportion of AIS A to B conversions in participants enrolled at 7-14 days would be 0.04 and 0.08 in the control and treatment groups, respectively. Similarly, the proportion of A to $\mathrm{C}$ (or better) conversions was assumed to be 0.03 for controls and 0.23 for the treatment group. Because this was an exploratory phase 2 trial, the power calculations utilized an Alpha of $<0.20$. With these assumptions, utilizing the Wilcoxon Rank Sum test, a sample of 34 participants in the treatment arm and 17 participants in the control arm provides $80 \%$ power for detecting significant effect. A dropout of up to 10 participants was assumed, raising the enrollment target to 61 .

\section{Statistical plan}

The a priori analysis plan for the primary outcome utilized a specific AIS improvement score with participants remaining AIS A assigned a score of ' 0 ', those improving from AIS A to AIS B assigned a score of ' 1 ', and those improving from AIS A to AIS C or better assigned a score of ' 2 '. The primary efficacy analysis compared the AIS improvement scores between the groups using the Wilcoxon Rank Sum test. The planned primary efficacy analysis was performed in two ways: the first utilizing all participants with at least 6 months of follow-up, the second analyzing only those participants for whom the primary efficacy endpoint was measured at the final 12-month time point. The $P$-value for these analyses of the primary outcome was set at 0.050 . The planned secondary efficacy analysis utilized Fisher's exact test for betweengroup comparisons of binary endpoints (for example, recovery yes/no). Analysis of changes from baseline in total motor and sensory scores was performed utilizing the $t$-test. The incidence of AEs was compared between the groups and analyzed for significance utilizing Fisher's exact test.

We certify that all applicable institutional and governmental regulations concerning the ethical use of human volunteers were followed during the course of this research.

\section{Amendments to the protocol}

There were several notable changes to the protocol during the course of the study.

1. The inclusion criterion for the rostral level of injury was changed from C5 neurological level to C5 motor level after several screen failures due to abnormal sensory testing at the C5 key point in participants with C5 motor level (and C4 sensory level). As the criterion was based on concerns related to the risk of motor level ascent, the change was intended to reduce the likelihood of screen failure while preserving the intended safety rationale.

2. After the first 12 participants had been enrolled (including 6 in the treatment group), the protocol was amended to increase the cell concentration prepared for loading into the surgical syringes. This change was prompted by analysis of treatment group syringes, which showed the calculated delivered dose was only $66 \%$ of the protocol-specified total dose of 1.5 million cells. Thus the first six treatment group participants were under-dosed compared with the subsequent 20 treatment group enrollees.

3. Just before the stoppage of the trial, the protocol was amended to go to a six syringe injection kit (one syringe filled with $20 \mu \mathrm{l}$ of cell suspension per injection site) to allow for easier assurance of injection volume consistency.

\section{Trial registration}

The trial was registered on clinicaltrials.gov (identifier NCT00073853).

\section{RESULTS}

\section{Recruitment and participant flow}

The trial commenced recruitment in October 2003 and concluded recruitment in March 2006 after 50 participants had been randomized. Recruitment was stopped by the sponsor short of the target enrollment owing to financial reasons that were not related to the trial. Active participants continued to be followed until October 2006 when, with funds completely depleted, the sponsor halted follow-up. A total of 10 participants ( 6 treatment and 4 control) reached the 6-month but not the final 12-month measurement point. In all, 8 of 
these 10 failures to achieve the 12-month follow-up visit were due to the sponsor's termination of study follow-up (5 in treatment group, 3 in control group); 1 (control group) was due to the participant leaving the country and one (treatment group) was for unknown reasons. Participant flow is described in Figure 1.

A detailed discussion of the recruitment pragmatics for this trial has previously been published. ${ }^{10}$ Participant demographics are displayed in Table 4. Comparisons of age, body mass index, neurological level (cervical vs thoracic), etiology, spine surgery, performance of spinal decompression/fusion, and the use of acute corticosteroid treatment did not show significant differences between the groups. The time from injury to macrophage injection for the treatment group participants was a mean of 12.93 days (median 13, mode 14 , range 9 to 17 ). There was only one exception to the 14-day macrophage injection window, a participant receiving macrophages at 17 days who had cell processing batch failure and was allowed to undergo repeat skin/blood harvest and processing, enabling macrophage injection as a protocol deviation.

\section{Primary outcome}

The complete efficacy analysis set $(n=43)$ was comprised of participants who had at least 6 months of follow-up. It included all those assigned to the control group $(n=17)$ and treatment group participants who received macrophage injection $(n=26)$. There were two planned analyses of the primary outcome: one on all participants followed for at least 6 months $(n=43)$ and the other restricted to those who had a full 12 months of follow-up data $(n=33)$. Table 5 summarizes the AIS outcomes. Although there was a trend favoring control, which was stronger in the $\geqslant 6$-month analysis set than the full 12-month analysis set, the results did not show a statistically significant difference between the groups. The proportion of participants in the full analysis set who converted from complete to incomplete neurological status was $26.9 \%$ in the treatment group and $58.8 \%$ in the control group. Of the 17 participants in the complete efficacy analysis set who were classified as incomplete at their last follow-up, 11 experienced 'anal-only' recoveries, leaving 6 that recovered more than ano-rectal sensation and/or anal contraction ( 2 in the treatment group, 4 in the control group). Of these, only 3 (1 treatment, 2 control) recovered any light touch or pin prick sensation in the L1 to S2 lower extremity dermatomes. Four participants improved to AIS C but none of them showed any recovery of lower extremity motor function-all were classified as AIS $\mathrm{C}$ only by virtue of anal contraction.

Several other post hoc analyses of the primary outcome were performed to further study the potential influence of other independent variables. To investigate the influence of injury level, the primary outcome was assessed separately for participants grouped by neurological level (cervical or thoracic). There was no significant difference between treatment vs control in either case (cervical $P=0.269$; thoracic $P=0.062$ ). The potential contribution of center effects was examined by analyzing the treatment vs control group differences for each of the study centers. The resultant $P$ values ranged from 0.20 to 0.74 , indicating that the primary outcome results were not statistically significant for any of the individual centers. We also examined the potential contribution of under-dosing, which occurred during the first 9 months of the study, by comparing the primary outcome analysis for participants enrolled before $(P=0.140)$ and after $(P=0.89)$ the protocol amendment, which increased the cell concentration prepared for loading into the surgical syringes. Although there was a trend favoring the control group in each of these post hoc

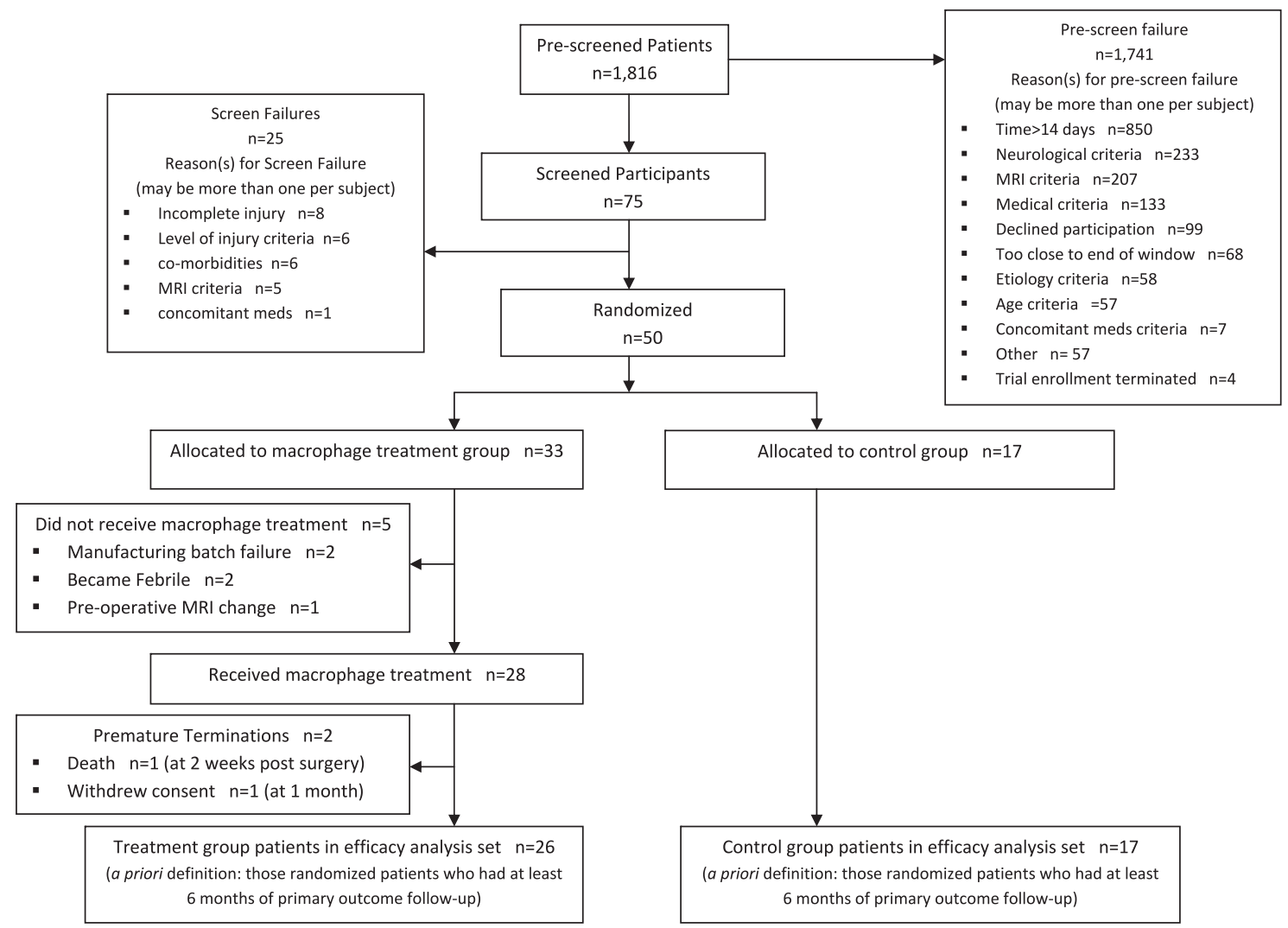

Figure 1 Participant Flow. 
Table 4 Participant demographics and clinical characteristics

\begin{tabular}{|c|c|c|c|c|}
\hline Variable & $\begin{array}{c}\text { All enrollees } \\
(n=50)\end{array}$ & $\begin{array}{l}\text { Treatment group } \\
\qquad(\mathrm{n}=33)\end{array}$ & $\begin{array}{l}\text { Control group } \\
\qquad(\mathrm{n}=17)\end{array}$ & P-value \\
\hline $\begin{array}{l}\text { Age } \\
\text { (range } \\
15^{\text {a }}-57 \text { years) }\end{array}$ & $\begin{array}{c}28.1 \pm 12.2 \\
\text { year }\end{array}$ & $\begin{array}{c}27.4 \pm 11.0 \\
\text { year }\end{array}$ & $\begin{array}{c}29.5 \pm 14.5 \\
\text { year }\end{array}$ & $0.57^{d}$ \\
\hline $\begin{array}{l}\text { BMI } \\
\text { (range } \\
17.30-36.52 \text { ) }\end{array}$ & $25.6 \pm 4.5$ & $25.8 \pm 4.9$ & $25.2 \pm 3.8$ & $0.66^{d}$ \\
\hline Gender & & & & \\
\hline $\begin{array}{l}\text { Male } \\
\text { Female }\end{array}$ & $\begin{array}{c}41 \\
9\end{array}$ & $\begin{array}{c}27 \\
6\end{array}$ & $\begin{array}{c}14 \\
3\end{array}$ & $1.00^{d}$ \\
\hline Neuro level & & & & \\
\hline $\begin{array}{l}\text { Cervical } \\
\text { Thoracic }\end{array}$ & $\begin{array}{l}24 \\
26\end{array}$ & $\begin{array}{l}15 \\
18\end{array}$ & $\begin{array}{l}9 \\
8\end{array}$ & $0.77^{e}$ \\
\hline Etiology & & & & \\
\hline $\begin{array}{l}\text { MVA } \\
\text { Fall } \\
\text { Sports }\end{array}$ & $\begin{array}{l}29 \\
11 \\
10\end{array}$ & $\begin{array}{c}20 \\
6 \\
7\end{array}$ & $\begin{array}{l}9 \\
5 \\
3\end{array}$ & $0.71^{\mathrm{e}}$ \\
\hline Spine surgery & & & & \\
\hline $\begin{array}{l}\text { Yes } \\
\text { No }\end{array}$ & $\begin{array}{c}47 \\
3\end{array}$ & $\begin{array}{c}31 \\
2\end{array}$ & $\begin{array}{c}16 \\
1\end{array}$ & $1.00^{\mathrm{e}}$ \\
\hline Decompression $^{\mathrm{C}}$ & & & & \\
\hline $\begin{array}{l}\text { Yes } \\
\text { No }\end{array}$ & $\begin{array}{l}33 \\
13\end{array}$ & $\begin{array}{c}22 \\
8\end{array}$ & $\begin{array}{c}11 \\
5\end{array}$ & $0.74^{e}$ \\
\hline Spine fusion & & & & \\
\hline $\begin{array}{l}\text { Yes } \\
\text { No }\end{array}$ & $\begin{array}{c}47 \\
3\end{array}$ & $\begin{array}{c}31 \\
2\end{array}$ & $\begin{array}{c}16 \\
1\end{array}$ & $1.00^{\mathrm{e}}$ \\
\hline Acute steroids ${ }^{c}$ & & & & \\
\hline $\begin{array}{l}\text { Yes } \\
\text { No }\end{array}$ & $\begin{array}{c}45 \\
3\end{array}$ & $\begin{array}{c}29 \\
2\end{array}$ & $\begin{array}{c}16 \\
1\end{array}$ & $1.00^{\mathrm{e}}$ \\
\hline
\end{tabular}

Abbreviations: BMI, body mass index; MVA, motor vehicle accident.

asubjects $<16$ years of age were approved by ethics committee before enrollment

bSurgery after injury but before trial enrollment.

'Data not available on all enrolled subjects.

${ }^{\mathrm{d}} t$-test with equal variance.

eFisher's exact test, 2-sided.

Table 5 Primary outcome results

\begin{tabular}{|c|c|c|c|c|c|c|}
\hline \multirow[t]{2}{*}{ AlS change } & \multicolumn{3}{|c|}{ At least 6 months $F / U(n=43)$} & \multicolumn{3}{|c|}{12 Months $F / U(n=33)$} \\
\hline & $\begin{array}{l}\text { Treatment } \\
(\mathrm{n}=26)(\%)\end{array}$ & $\begin{array}{l}\text { Control } \\
(\mathrm{n}=17)(\%)\end{array}$ & & $\begin{array}{l}\text { Treatment } \\
(\mathrm{n}=19)(\%)\end{array}$ & $\begin{array}{l}\text { Control } \\
(\mathrm{n}=14)(\%)\end{array}$ & \\
\hline AIS $A \rightarrow A$ & $19(73)$ & $7(41)$ & & $14(74)$ & $6(43)$ & \\
\hline AIS $A \rightarrow B$ & $5(19)$ & $8(47)$ & & $4(21)$ & $7(50)$ & \\
\hline AIS $A \rightarrow C$ & $2(8)$ & $2(12)$ & & $1(5)$ & $1(7)$ & \\
\hline $\begin{array}{l}\text { Total group } \\
\text { AIS change } \\
\text { score }^{a}\end{array}$ & 9 & 12 & $P=0.053^{b}$ & 6 & 9 & $P=0.095^{b}$ \\
\hline
\end{tabular}

Abbreviation: AIS, ASIA Impairment Scale.

${ }^{a}$ AIS change score: $A \rightarrow A$ patients assigned $O$ points; $A \rightarrow B$ patients assigned 1 point; $A \rightarrow C$ or better patients assigned two points (there were no patients with AIS D or AIS E recovery).

bWilcoxon Rank Sum test.

analyses, in none of them was the difference between the groups significant.

\section{Secondary outcomes}

The motor and sensory secondary outcomes are shown in Table 6. The analyses failed to show significant differences between the groups in the motor outcomes. Although the mean total motor score change was slightly greater in the control group and the proportion of cervical participants that experienced a two motor level improvement was greater in the treatment group, in neither case was the difference significant. Sensory outcomes showed significantly greater light touch score improvement in the control group and a strong trend favoring the control group in pin prick score improvement. A higher proportion of the treatment group participants showed a two sensory level improvement compared with the controls but the difference was not statistically significant. Analysis of the mean upper extremity motor score change in the cervical patients failed to show a significant difference between the groups. Similarly, there was no difference in lower extremity motor score change between the groups for thoracic patients. Analysis of sensory/motor level deterioration in the cervical participants showed 4 ( 3 active and 1 control) who lost one motor level unilaterally-the between-group difference was not significant $(P=0.381)$. There were two cervical participants (both in the control group) who lost one sensory level unilaterally but the between-group difference was not significant $(P=0.108)$.

The bowel and bladder outcomes are shown in Table 7. The proportion of participants reporting recovery of some bladder and bowel awareness was greater in the control group but the difference was not significant. None of the study participants experienced recovery of volitional bowel or bladder control.

\section{Exploratory outcomes}

The trial included exploratory measures of activities (FIM), participation (CHART) and quality of life (SWLS) in an attempt to broaden the range of assessments beyond measures of impairment. Betweengroup differences were analyzed for final FIM and FIM change as well as the final CHART (including the Physical Independence and Mobility Subscales) and SWLS. There were no significant differences between the groups in FIM, CHART or SWLS outcomes as shown in Table 8.

\section{Safety Outcomes}

The safety analysis set $(n=44)$ was comprised of all participants who were still in the trial at Day 0 of the protocol when the treatment group was scheduled to have macrophage injection. Treatment group participants in the $\mathrm{AE}$ analysis set $(n=27)$ included all those who underwent macrophage injection except for one whose AE data were lost after completion of the trial. In addition, this group included data from a participant who died 41 days after macrophage injection and one of the participants who did not receive treatment because of macrophage manufacturing batch failure but was followed for collection of safety data. Safety was assessed by analysis of the incidence of AEs abstracted from the clinical record and unstructured follow-up interviews that occurred during the study period as shown in Table 9. This listing of AEs includes those that were experienced by at least $10 \%$ of the participants and is representative of complications commonly encountered in patients treated for acute SCI. Pain events were categorized by location (for example, spine, extremity) and by neuropathic vs musculoskeletal but not by severity. Between-group comparisons of individual AEs showed no significant differences. The mean number of reported AEs was 31.39 (95\% CI 24.42, 38.35) for the treatment group compared with 31.00 (95\% CI 22.58, 39.46) for the control group $(P=0.942)$.

There were 15 serious AEs (SAEs)(10 treatment group, 5 control group) recorded for 10 participants during the trial as shown in Table 10. Nine of these were deemed to be unrelated to trial participation by the investigators, two were felt unlikely to be related. 
Table 6 Secondary outcomes: motor and sensory

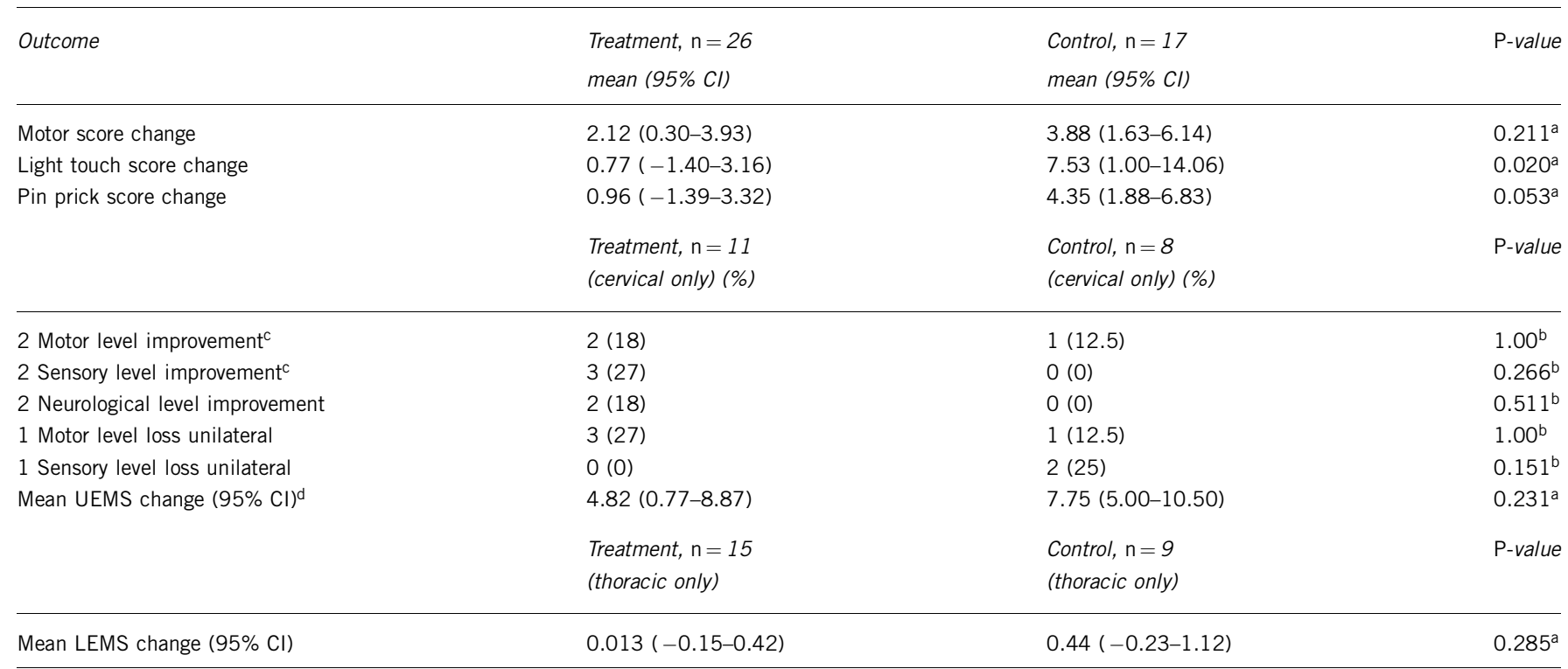

Abbreviations: LEMS, lower extremity motor score; UEMS, upper extremity motor score.

at-test.

bFisher's Exact Test (two sided).

${ }^{\mathrm{C} A t}$ least unilateral 2-level improvement.

dThere was no LEMS change in the cervical patients: UEMS change $=$ TMS change.

Table 7 Secondary outcomes: bowel and bladder

\begin{tabular}{llll}
\hline Outcome & $\begin{array}{l}\text { Treatment, } \\
\mathrm{n}=26(\%)\end{array}$ & $\begin{array}{l}\text { Control, } \\
\mathrm{n}=17(\%)\end{array}$ & P-value \\
\hline Awareness of need to void/empty bladder & $6(23)$ & $8(47)$ & 0.182 \\
Volitional controlled micturition & 0 & 0 & \\
Awareness of need to evacuate bowels & $9(35)$ & $8(47)$ & 0.528 \\
Volitional controlled bowel evacuation & 0 & 0 &
\end{tabular}

aFisher's exact test (two sided)

In two participants, the SAEs were felt to be probably related to trial participation. In one instance, a participant with a prior cervical spine fusion who had undergone laminectomy for macrophage injection was diagnosed with late spinal instability 3 months after treatment, prompting a one-segment extension of the prior anterior fusion and addition of a posterior lateral mass fusion. The prior laminectomy was felt to be a contributing factor in the development of subsequent spinal instability. Follow-up assessment demonstrated spinal stability. In the other instance, a participant with C5 tetraplegia who underwent macrophage injection developed significant subsegmental atelectasis on the second post-operative day requiring intensive care unit transfer for bronchoscopy. The participant responded well to treatment and was returned to the ward with atelectasis resolved within 2 days.

One participant developed bacterial meningitis with Enterobacter cloacae diagnosed on protocol-specified cerebrospinal fluid (CSF) culture 7 days after macrophage implantation. Sterility tests on the macrophage injection product were negative and the participant responded well to antibiotic treatment with follow-up CSF cultures showing no bacterial growth. A pre-existing dural tear and CSF leak had been noted at the time of the macrophage implantation surgery, raising the possibility of surgical infection either at the time of initial cervical spine stabilization or subsequent macrophage implantation. Five weeks after macrophage implantation, this same participant developed significant neck pain, prompting a work-up, which showed a pseudomeningocele requiring surgical exploration and repair of persistent CSF leak. This participant's SAEs were felt to be possibly related to trial treatment group assignment by the investigator.

There was one death during the trial in a participant with C6 tetraplegia who was in the treatment group. Although eligibility criteria were met, it was noted that the individual was obese, with a body mass index of $38.2 \mathrm{~kg} \mathrm{~m}^{-2}$. The clinical course following macrophage injection was unremarkable except for Clostridium difficile colitis until the 14th postoperative day when the participant developed onset of sepsis and respiratory failure due to bronchopneumonia requiring intubation and mechanical ventilation. The participant went on to develop multisystem failure and died after life support treatment was withdrawn on the 41st postoperative day. The investigator determined that the SAE was unlikely related to participation in the trial. The postmortem examination diagnosis attributed the cause of death to be complications of tetraplegia due to cervical SCI.

\section{DISCUSSION}

On the basis of the phase 1 trial experience, it was hypothesized that this protocol for autologous incubated macrophage treatment of participants with acute complete SCI would be associated with improved neurological outcomes. The phase 2 study we report here failed to support this hypothesis. Although there was a trend noted in the primary outcome measurement favoring the control group, neither of the planned assessments met criteria for statistical significance, and as such, the null hypothesis cannot be rejected.

Perhaps, the most striking finding of this study was the extraordinary conversion rate in the control group in which 10 of the 17 
Table 8 Exploratory outcomes: activities, participation and quality of life

\begin{tabular}{llll}
\hline Outcome & $\begin{array}{l}\text { Treatment, } \mathrm{n}=26 \\
\text { mean }(95 \% \mathrm{Cl})\end{array}$ & $\begin{array}{l}\text { Control, } \mathrm{n}=17 \\
\text { mean }(95 \% \text { Cl) }\end{array}$ \\
\hline Final FIM $^{\mathrm{a}}$ & $64.12(56.72-71.51)$ & $64.24(52.98-75.49)$ & 0.985 \\
FIM change $^{\mathrm{a}}$ & $42.88(35.89-49.88)$ & $37.24(27.64-46.83)$ & 0.316 \\
Total CHART score $^{\mathrm{b}}$ & $497.96(465.19-530.73)$ & $524.35(489.09-559.62)$ & 0.275 \\
CHART physical independence $^{\mathrm{b}}$ & $94.67(92.00-97.35)$ & $94.12(88.69-99.54)$ & 0.739 \\
CHART mobility $^{\mathrm{b}}$ & $85.77(79.40-92.14)$ & $90.94(85.00-96.88)$ & 0.252 \\
Satisfaction with life scale $^{\mathrm{b}}$ & $19.12(15.33-22.90)$ & $22.59(18.77-26.41)$ & 0.206 \\
\hline
\end{tabular}

Abbreviations: FIM, functional independence measure; CHART, craig handicap assessment and reporting technique.

aChange in Motor FIM between day 7 post-op and last F/U at 6 or 12 months.

bTotal CHART and subscales and SWLS at last F/U visit at 6 or 12 months.

${ }^{\mathrm{c}} \mathrm{t}$-test.

Table 9 Adverse events

\begin{tabular}{|c|c|c|c|}
\hline $\begin{array}{l}\text { Adverse events experienced } \\
\text { by } \geqslant 10 \% \text { of patients }\end{array}$ & $\begin{array}{l}\text { Treatment, } \\
\mathrm{n}=27^{\mathrm{a}}(\%)\end{array}$ & $\begin{array}{l}\text { Control, } \\
\mathrm{n}=17^{\mathrm{a}}(\%)\end{array}$ & $\mathrm{P}$-value ${ }^{\mathrm{b}}$ \\
\hline UTI & $24(89)$ & $15(88)$ & 1.000 \\
\hline Spine pain & $19(70)$ & $10(59)$ & 0.521 \\
\hline Insomnia & $16(59)$ & $13(76)$ & 0.333 \\
\hline Anemia & $18(67)$ & $11(65)$ & 1.000 \\
\hline Spasticity & $15(56$ & $12(71$ & 0.360 \\
\hline Constipation & $15(56$ & $11(65)$ & 0.754 \\
\hline Pain (NOS) & $13(48$ & $11(65)$ & 0.359 \\
\hline Neuropathic pain & $14(56$ & $9(53)$ & 1.000 \\
\hline Pyrexia & $16(59)$ & $8(47)$ & 0.539 \\
\hline Nausea & $13(48$ & $9(53)$ & 1.000 \\
\hline Skin sore & $13(48$ & $9(53$ & 1.000 \\
\hline Joint pain & $11(41)$ & $7(41)$ & 1.000 \\
\hline Headache & $12(44)$ & $7(41)$ & 1.000 \\
\hline Anxiety & $10(37)$ & $5(29)$ & 0.748 \\
\hline Orthostatic hypotension & $10(37)$ & $6(35$ & 1.000 \\
\hline Depression & $10(37)$ & $6(35)$ & 1.000 \\
\hline Heterotopic ossification & $8(30$ & $7(41)$ & 0.521 \\
\hline Extremity pain & $7(26)$ & $6(35)$ & 0.521 \\
\hline Rash & $9(33)$ & $4(24)$ & 0.735 \\
\hline Peripheral edema & $6(22)$ & $4(24)$ & 1.000 \\
\hline Abdominal pain & $7(26)$ & $3(18)$ & 0.716 \\
\hline Pruritis & $5(19)$ & $4(24)$ & 0.716 \\
\hline Deep vein thrombosis & $4(15)$ & $5(29)$ & 0.275 \\
\hline Fungal infection & $6(22)$ & $2(12)$ & 0.455 \\
\hline Hypokalemia & $6(22)$ & $2(12)$ & 0.455 \\
\hline Upper respiratory infection & $2(7)$ & $5(29)$ & 0.089 \\
\hline Atelectasis & $5(19)$ & $2(12)$ & 0.689 \\
\hline C. difficile colitis & $4(15)$ & $3(18)$ & 1.000 \\
\hline Dysautonomia & $4(15)$ & $3(18)$ & 1.000 \\
\hline Liver enzyme elevation & $4(15)$ & $3(18)$ & 1.000 \\
\hline Emesis & $4(15)$ & $3(18)$ & 1.000 \\
\hline Dehydration & $3(11)$ & $3(18)$ & 0.662 \\
\hline Fecal incontinence/diarrhea & $4(15)$ & $7(41)$ & 0.089 \\
\hline Musculoskeletal pain & $3(11)$ & $3(18)$ & 0.662 \\
\hline Pneumonia & $3(11)$ & $3(18)$ & 0.662 \\
\hline Staphylococcus infection & $2(7 \%)$ & $3(18)$ & 0.359 \\
\hline Hypoalbuminemia & $5(19)$ & $0(0)$ & 0.139 \\
\hline Abdominal distension & $3(11)$ & $1(6)$ & 1.000 \\
\hline Bladder calculus & $1(4)$ & $3(18)$ & 0.282 \\
\hline Cellulitis & $3(11)$ & $1(6)$ & 1.000 \\
\hline
\end{tabular}

Abbreviation: NOS, not otherwise specified.

'Data set includes subjects that were still in the study at 'day 0' macrophage implant time point. AE data was not available on one treatment group patient.

bFisher's exact test (two-sided) participants $(58.8 \%)$ converted from complete to incomplete injury status over the 6-12 month course of follow-up. Recent published analyses of existing SCI databases have typically found such conversion rates to be in the $20-30 \%$ range, ${ }^{14,15}$ which is similar to that found in our treatment group in which 7 of 26 (26.9\%) experienced conversions to incomplete status. Although it is certainly possible that the high conversion rate in the control group is a 'true' representation of spontaneous recovery in the SCI population, clinical experience and published reports would suggest otherwise, prompting consideration of the possibility that the conversion rate was anomaly associated with the relatively small size of the group (the result of the overall small size of the trial further diminished by the 2:1 randomization scheme).

Regardless, the interpretation of the results of the trial must rest on the head-to-head primary outcome comparison between the groups, and in this regard, we cannot recommend macrophage treatment of acute SCI as specified in this protocol. The secondary outcome measurements of index score change favored control group but only the light touch score change was significant. The secondary measures of sensory/motor level improvement, although more commonly achieved in the treatment group, were small in number and not significant. Measurement of bowel and bladder functional recovery similarly failed to show significant differences between the groups, either in terms of recovery of volitional control, which none of the participants achieved, or in terms of sensory awareness. Thus, from the perspective of efficacy, this trial failed to deliver on the experience of the phase 1 results. In this context, it should be noted that the protocol was significantly modified between phase 1 (which was conducted at a single center) and the phase 2 multicenter trial reported here. Perhaps most importantly there was a significant change in dose between the two trials. On the basis of unpublished animal studies performed concurrently with phase 1 investigating cell distribution after spinal cord injection of macrophages, the treatment regimen was changed from a total dose of 4 million cells delivered in four injections in phase 1 to 1.5 million cells in six injections in phase 2. These studies showed that the optimal dose was 250000 cells per injection and that the migration of the cells was limited, prompting an increase in the number of injections. This change was intended to more effectively distribute the cell treatment across the caudal contusion boundary, targeting the region of the posterior columns, the corticospinal tracts and the spinothalamic tracts but resulted in a significant reduction in the total dose. Furthermore, the delivered dose in the first six treatment group patients in this trial was found to be only $66 \%$ of the intended number of cells. Although analysis of the primary outcome comparing participants enrolled in the 'under-dosed' and 'fully dosed' eras of the trial failed to show 
Table 10 Serious adverse events

\begin{tabular}{llll}
\hline Serious adverse event & Patients & Group & $\begin{array}{l}\text { Relationship } \\
\text { to Treatment }\end{array}$ \\
\hline Pedicle screw dislodged & 1 & Control & Not related \\
Surgery for pressure sore & 3 & Control (3) & Not related \\
Hospitalization for urosepsis & 3 & Control (1), & Not related \\
& & treatment (2) & \\
Hospitalized for cellulitis & 1 & Treatment & Not related \\
Surgery for late spinal instability & 1 & Treatment & Probably related \\
Post-op subsegmental atelectasis & 1 & Treatment & Probably related \\
Death due to multi-organ failure & 1 & Treatment & Unlikely \\
Lower extremity DVT & 1 & Treatment & Not related \\
Surgery for scrotal infection & 1 & Treatment & Unlikely \\
Bacterial meningitis & 1 & Treatment & Possibly related \\
Pseudomeningocele & 1 & Treatment & Possibly related
\end{tabular}

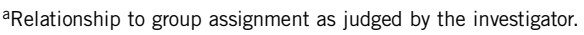

significant differences, the experience of this study reinforces the need for attention to optimal dosing, including its preclinical derivation with appropriate scale-up calculations, and in the confirmation of its delivery during the trial. Additionally, in contrast to the phase 1 trial, the primary outcome examiners and the investigators responsible for data interpretation (classification of AIS grade and neurological/ motor/sensory level) were formally trained and tested for reliability at the outset of this study, presumably reducing the likelihood of bias or measurement error.

It should also be acknowledged that independent variables other than the macrophage intervention may have had a role in the outcome of the trial. Although there were extensive meetings of the surgeon investigators to discuss surgical variables and standardization of the macrophage injection protocol, decisions regarding neurosurgical technique such as the use of intraoperative spinal sonography for targeting, expansion duraplasty, and additional decompression were left to the clinical judgment of the surgeon. Although the study did not collect data that would allow analysis of these and other details of surgical technique to the extent that differences in approach tend to be associated with study center (in all but one center, there was a single surgeon investigator), the lack of significant center effect suggests that such surgical variables did not significantly impact the outcome of the study. Nonetheless, it is recommended that future trials of surgical interventions standardize technique variables to the extent possible and collect data on those elements that cannot be standardized to enable analysis of key surgical variables. The injection technique-use of a hand-held syringe-itself may be a confounding variable as well as a potential source of tissue damage. These concerns prompted suggestions for development of a more sophisticated standardized injection methodology with flexible tubing separating the needle from the syringe and a hydraulic injection system to enable precise calibration of volume and rate.

The rehabilitation process, both what was provided by clinicians and the effort expended by participants, may also have a role in the outcomes measured. Although we did not measure the quantity and intensity of rehabilitation treatments nor the motivation and effort of the participants, we found no reason to suspect a systematic bias that favored one group over the other. Indeed, the FIM and CHART variables, which would be expected to reflect such bias and provide the best measure of rehabilitation outcomes, showed no differences between the groups. With specific reference to the primary outcome, there is currently no compelling evidence that rehabilitation or patient effort can improve the likelihood of AIS conversion. It could be argued that strengthening exercises, if unequally delivered to the groups might result in differences in the secondary outcome of motor level or upper extremity motor score change, but no significant differences were found between the groups in these measures.

Exploratory post hoc analyses also provided some insights regarding the efficacy outcome measures themselves, specifically with regard to the functional impact of AIS conversion. In this study, there were 13 participants who improved from AIS A to B and an additional 4 who improved to AIS C. Of those who regained sufficient sensory/ motor function to be classified as incomplete, most converted purely on the basis of some combination of S4-5 sensation, ano-rectal sensation, and/or anal contraction. Only three of the incomplete participants regained any sensation in the lower extremity dermatomes L1-S3. Of those three, only one regained more than 2 of the possible 48 sensory index (Light Touch or Pin Prick) points in the lower extremity dermatomes from L1 to S3. Furthermore, none of those who improved to AIS C recovered any motor function in their lower extremities. Thus, although primary efficacy of the trial was based on AIS conversion, the functional impact of conversion in terms of improved lower extremity sensory/motor function was negligible. This finding should give pause to those who might consider use of AIS conversion as currently defined in the ISNCSCI as an outcome measure in future interventional trials. Indeed, the utility of AIS conversion as a clinical trial endpoint has been questioned previously. ${ }^{16}$

Safety was assessed by analysis of AEs and SAEs. In spite of protocol requirements for two surgeries (skin harvest and macrophage injection), we did not find significant safety concerns in the analysis of AEs. Interestingly, although the sequence of protocol surgeries required a several day interruption of anticoagulant venous thromboembolism prophylaxis (for example, low molecular weight heparin), we did not find a significant difference of thrombotic complications between the groups. Review of the 15 SAEs showed that the majority (9) were felt by the investigators to be 'unrelated' to treatment group assignment. The four SAEs noted to be 'probably' or 'possibly' related to treatment group assignment included postoperative atelectasis, late spinal instability, bacterial meningitis, and pseudomeningocele, all serving as a reminder that the safety considerations of cell-based therapies include the method of delivery-in this case, surgery on the spinal cord requiring laminectomy and opening of the dura.

The single death in the study was determined to be due to complications of traumatic tetraplegia. This occurrence is indicative of the fragile physiological status of acute SCI patients, reinforcing the importance of safety considerations in the determination of eligibility. In this study, although safety concerns were inherent in a number of the criteria, significant obesity was not a specific criterion for exclusion. Although obesity in persons with SCI is garnering increasing scientific scrutiny, correlations with mortality risk and risk of surgical complications have not been studied. ${ }^{17}$ Furthermore, surgical outcome studies have not established clear association with increased early mortality but have suggested a possible influence of obesity on complication risk and length of stay. ${ }^{18,19}$ Although it would be difficult at present to define an evidence-based threshold criterion for an obesity exclusion in SCI trials, further research in this area might justify such consideration. 


\section{Study limitations}

The ability to generalize the results of this study is limited by the small number of enrolled participants. Not only was the projected enrollment based on power calculations for a phase 2 trial, but actual enrollment fell short owing to the premature termination of the project. The eligibility criteria must also be considered-many patients were excluded from participation and the results must be interpreted in the context of the subset of eligible patients. Specifically, enrollment in this trial was limited to patients with acute complete SCI within a defined range of neurological levels who had $\leqslant 3 \mathrm{~cm}$ MRI-measured contusions. Additionally, the protocol did not specify or otherwise attempt to strictly control or measure plausible independent variables such as acute treatment with high-dose steroids or the details of ancillary surgical procedures such as duraplasty and intraoperative use of ultrasound for treatment targeting. It should also be noted that the time from injury to macrophage injection was clustered at the high end of the 14-day window, limiting the ability to assess efficacy and safety for treatment delivered at earlier time frames. The rehabilitation variable, while referenced in the protocol to accomplishment of accepted functional outcomes, ${ }^{12}$ was not effectively standardized or measured with respect to specific treatment modalities, intensity or duration of treatment.

\section{Future Research}

Although results presented here do not provide support for further clinical trials of autologous macrophage treatment for SCI, this failure to show positive results should be viewed in the context of the protocol-the manufacturing process, number of cells, and route of administration-rather than in the broader context of the underlying validity of immune modulation as a therapeutic approach. Indeed, promising preclinical research has been published since the conclusion of this trial, which may lead to clinical translation in the future. Recent reports have shown that a specific subset of blood-derived macrophages with properties similar to the cells utilized in our study appear to have an anti-inflammatory, regeneration-promoting activity in animal models of SCI. ${ }^{20,21,22}$ It may be possible with further advances in this area of research to more effectively manipulate the natural process of macrophage activation resulting in production of an improved therapeutic macrophage phenotype. Importantly, these studies also suggest the possibility of alternative and less invasive methods of cell delivery (blood stream, CSF) that warrant further development. $^{22}$

The experience of this study should also provide lessons for future clinical trials with regard to the importance of standardization and measurement of key practice parameters such as surgical techniques and rehabilitation that may be important independent variables.

\section{CONCLUSIONS}

This study reports the results of the first multicenter RCT of a cell therapy for patients with acute SCI. Although there was a trend favoring the standard of care control group, the analysis failed to show a significant difference in the primary outcome between those participants who received autologous incubated macrophage treatment and those who did not. Sensory index scores showed more improvement in the control group participants but there was no significant difference in motor index scores between the groups, nor was there a difference in the exploratory measures of FIM, CHART or SWLS. There was no difference in the incidence of AEs between the groups but several of the SAEs were probably related to treatment group assignment. Although these results do not support treatment of complete SCI with autologous incubated macrophage therapy as specified in this protocol, it is hoped that ongoing preclinical research will yield improved methods of producing a therapeutic cell phenotype and less invasive cell delivery.

\section{DATA ARCHIVING}

There were no data to deposit.

\section{CONFLICT OF INTEREST}

The authors declare no conflict of interest.

\section{ACKNOWLEDGEMENTS}

We wish to thank the patients who volunteered to participate in this research; Gale Whiteneck and Chris Pretz of the Craig Hospital Research Department for their guidance in the analysis of data; Michal Schwarz and Eti Yoles for their helpful reviews of the manuscript; and Craig Hospital for funding the analysis. This study was sponsored by Proneuron Biotechnologies, Rehovot, Israel. ClinicalTrials.gov identifier NCT00073853.

1 American Association of Neurological Surgeons/Congress of Neurological Surgeons. Pharmacological therapy after acute cervical spinal cord injury. Neurosurgery 2002 50: S63-S72.

2 Rasouli A, Lammertse DP, Wang MY. A review of clinical trials and future directions for clinical studies in acute spinal cord injury. In: Lin VW (ed.) Spinal Cord Medicine: Principles and Practice, 2nd edn. Demos Medical: New York, 2010, pp 922-929.

3 Hawryluk GW, Rowland J, Kwon BK, Fehlings MG. Protection and repair of the injured spinal cord: a review of completed, ongoing, and planned clinical trials for acute spinal cord injury. Neurosurg Focus 2008; 5: 1-16.

4 Perry VH, Brown MC, Gordon S. The macrophage response to central and periphera nerve injury. A possible role for macrophages in regeneration. J Exper Med 1987; 165 1218-1223.

5 Zeev-Brann AB, Lazarov-Spiegler O, Brenner T, Schwartz M. Differential effects of central and peripheral nerves on macrophages an microglia. Glia 1998. 23: 181-190.

6 Lazarov-Spiegler 0, Solomon AS, Schwartz M. Peripheral nerve-stimulated macrophages simulate a peripheral nerve-like regenerative response in rat transected optic nerve. Glia 1998; 24: 329-337.

7 Rapalino O, Lazarov-Spiegler O, Agranov E, Velan GJ, Yoles E, Fraidakis M et al. Implantation of stimulated homologous macrophages results in partial recovery of paraplegic rats. Nat Med 1998; 4: 814-821.

8 Bromstein Y, Marder JB, Vitner K, Smirnov I, Lisaey G, Butovsky 0 et al. Features of skin-coincubated macrophages that promote recovery from spinal cord injury. J Neuroimmunol 2003; 142: 10-16.

9 Knoller N, Auerbach G, Fulga V, Zelig G, Attias J, Bakimer R et al. Clinical experience using incubated autologous macrophages as a treatment for complete spinal cord injury: Phase I study results. J Neurosurg Spine 2005; 3: 173-181.

10 Jones LAT, Lammertse DP, Charlifue SB, Kirshblum SC, Apple DF, Ragnarsson KT et al. A phase 2 autologous cellular therapy trial in patients with acute, complete spinal cord injury: pragmatics, recruitment, and demographics. Spinal Cord 2010; 48 798-807.

11 American Spinal Injury Association. International Standards for Neurological Classification of Spinal Cord Injury. revised 2000, reprinted 2002 American Spinal Injury Association Chicago, IL, 2002.

12 Consortium for Spinal Cord Medicine. Outcomes Following Traumatic Spinal Cord Injury: Clinical Practice Guidelines for Health-care Professionals. Paralyzed Veterans of America: Washington, DC, 1999.

13 Marino RJ, Jones L, Kirshblum S, Tal J, Dasgupta A. Reliability and repeatability of the motor and sensory examination of the International Standards for Neurological Classification of Spinal Cord Injury. J Spinal Cord Med 2008; 31: $166-170$

14 Fawcett JW, Curt A, Steeves JD, Coleman WP, Tuszynski MH, Lammertse DP et al. Guidelines for the conduct of clinical trials for spinal cord injury as developed by the ICCP panel: spontaneous recovery after spinal cord injury and statistical power needed for therapeutic clinical trials. Spinal Cord 2007; 45: 190-205.

15 Spiess M, Müller RM, Rupp R, Schuld C. van Hedel HJA. Conversion in ASIA impairment scale during the first year after traumatic spinal cord injury. J Neurotrauma 2009; 26: 2027-2036.

16 Steeves JD, Lammertse D, Curt A, Fawcett JW, Tuszynski MH, Ditunno JF et al Guidelines for the conduct of clinical trials in spinal cord injury as developed by the ICCP panel: clinical trial outcome measures. Spinal Cord 2007; 45: 206-221.

17 Rajan S, McNeely MJ, Warms C, Goldstein B. Clinical assessment and management of obesity in individuals with spinal cord injury: a review. J Spinal Cord Med 2008; 31 361-372. 
18 Choban PS, Flancbaum L. The impact of obesity on surgical outcomes: a review. J Am Coll Surg 1997; 185: 593-603.

19 Patel N, Bagan B, Vadera S, Maltenfort MG, Deutsch H, Vaccaro AR et al. Obesity and spine surgery: relation to perioperative complications. J Neurosurg Spine 2007; 6: 291-297.

20 Shechter R, London A, Varol C, Raposo C, Cusimano M, Yovel G et al. Infiltrating bloodderived macrophages are vital cells playing an anti-inflammatory role in recovery from spinal cord injury in mice. PLoS Med 2009; 6: e1000113.
21 Kigerl KA, Gensel JC, Ankeny DP, Alexander JK, Donnelly DJ, Popovich PG. Identification of two distinct macrophage subsets with divergent effects causing either neurotoxicity or regeneration in the injured mouse spinal cord. J Neuroscience 2009; 29: $13435-13444$.

22 Schwartz M. 'Tissue-repairing' blood-derived macrophages are essential for healing of the injured spinal cord: from skin-activated macrophages to infiltrating blood-derived cells? Brain Behav Immun 2010; 24: 1054-1057. 